\title{
Integrated microdevice with a windmill-like hole array for the clog-free, efficient, and self-mixing enrichment of circulating tumor cells
}

\author{
Hao Li ${ }^{1,2}$, Jinze Li ${ }^{2}$, Zhiqi Zhang ${ }^{2}$, Zhen Guo ${ }^{1,2,3}$, Changsong Zhang ${ }^{4}$, Zixu Wang ${ }^{2}$, Qiuquan Guo ${ }^{5}$, Chao Li

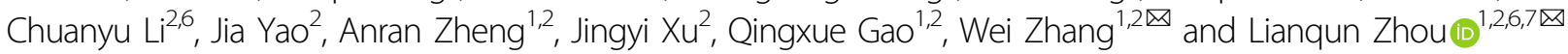

\begin{abstract}
Circulating tumor cells (CTCs) have tremendous potential to indicate disease progression and monitor therapeutic response using minimally invasive approaches. Considering the limitations of affinity strategies based on their cost, effectiveness, and simplicity, size-based enrichment methods that involve low-cost, label-free, and relatively simple protocols have been further promoted. Nevertheless, the key challenges of these methods are clogging issues and cell aggregation, which reduce the recovery rates and purity. Inspired by the natural phenomenon that the airflow around a windmill is disturbed, in this study, a windmill-like hole array on the SU-8 membrane was designed to perturb the fluid such that cells in a fluid would be able to self-mix and that the pressure acting on cells or the membrane would be dispersed to allow a greater velocity. In addition, based on the advantages of fluid coatings, a lipid coating was used to modify the membrane surface to prevent cell aggregation and clogging of the holes. Under the optimal conditions, recovery rates of $93 \%$ and $90 \%$ were found for A549 and HeLa cells in a clinical simulation test of our platform with a CTC concentration of 20-100 cells per milliliter of blood. The white blood cell (WBC) depletion rate was $98.7 \%(n=15)$, and the CTC detection limit was less than 10 cells per milliliter of blood $(n=6)$. Moreover, compared with conventional membrane filtration, the advantages of the proposed device for the rapid $(2 \mathrm{~mL} / \mathrm{min})$ and efficient enrichment of CTCs without clogging were shown both experimentally and theoretically. Due to its advantages in the efficient, rapid, uniform, and clog-free enrichment of CTCS, our platform offers great potential for metastatic detection and therapy analyses.
\end{abstract}

\section{Introduction}

Circulating tumor cells (CTCs), which are shed from a primary tumor and released into the bloodstream, play a seed-like role in the process of cancer metastasis and cause as much as $90 \%$ of the cancer-associated morta lity ${ }^{1-3}$. The possibility of isolating CTCs from peripheral blood makes it possible for CTCs to serve as a "liquid

\footnotetext{
Correspondence: Wei Zhang (zhangw@sibet.ac.cn) or

Lianqun Zhou (zhoulq@sibet.ac.cn)

${ }^{1}$ School of Biomedical Engineering (Suzhou), Division of Life Sciences and

Medicine, University of Science and Technology of China, 260026 Hefei, China

${ }^{2}$ CAS Key Lab of Bio-Medical Diagnostics, Suzhou Institute of Biomedical

Engineering and Technology, Chinese Academy of Sciences, 215163 Suzhou, China

Full list of author information is available at the end of the article
}

biopsy" for metastatic tumors and provide cancer-related information in a noninvasive manner ${ }^{4-6}$. Clinical studies have shown that the CTC counts or number of cancer patients is correlated with disease, which can indicate disease progression and evaluate the therapeutic response $\mathrm{e}^{7,8}$. Based on abundant intrinsic information highly associated with cancer metastasis, the isolation and detection of CTCs is valuable in both clinical research and treatment. Therefore, the development of a new platform is vital and urgent for making the isolation and detection of CTCs more rapid, efficient, and accurate.

Currently, due to the extreme rarity and high heterogeneity of CTCs as well as their vulnerability, the isolation and detection CTCs from whole blood are extremely

\section{(c) The Author(s) 2022}

(c) (i) Open Access This article is licensed under a Creative Commons Attribution 4.0 International License, which permits use, sharing, adaptation, distribution and reproduction in any medium or format, as long as you give appropriate credit to the original author(s) and the source, provide a link to the Creative Commons license, and indicate if changes were made. The images or other third party material in this article are included in the article's Creative Commons license, unless indicated otherwise in a credit line to the material. If material is not included in the article's Creative Commons license and your intended use is not permitted by statutory regulation or exceeds the permitted use, you will need to obtain permission directly from the copyright holder. To view a copy of this license, visit http://creativecommons.org/licenses/by/4.0/. 
challenging ${ }^{9,10}$. Various techniques that are mostly based on specific tumor markers (affinity-based strategies) and biophysical properties (label-free strategies) have been developed for the effective isolation and detection of $\mathrm{CTCs}^{9}$. Affinity-based capture depends on the immunochemical interactions between specific antigens expressed on the cell surface and their corresponding antibodies immobilized on magnetic beads ${ }^{11-13}$ or patterned structures $^{14-18}$. To date, CellSearch, the only Food and Drug Administration (FDA)-approved platform for CTC detection in breast, prostate and colorectal cancer, has been the gold standard in the field of $\mathrm{CTCs}^{7,19,20}$. Nevertheless, due to loss of the epithelial phenotype related to epithelial-mesenchymal transition (EMT) during tumor metastasis ${ }^{21}$, the effect of CTC capture based on epithelial cell adhesion molecular (EpCAM) expression, including CellSearch, has been questioned. In contrast, label-free strategies, with their unique advantages of easy operations and low costs, can capture epithelial and mesenchymal phenotypes, which are more suitable for the critical requirements for clinical application.

There are numerous approaches for CTC capture depending on biophysical properties, such as size and deformability ${ }^{10,22,23}$, electrochemical properties ${ }^{24,25}$, dielectrophoretic separation ${ }^{26,27}$, inertial migration ${ }^{28,29}$, and other characteristics. Nonetheless, among these characteristics, membrane filtration, as a simpler and lowercost technique based on the difference in size between tumor cells and hematologic cells, has prompted significant interest $\mathrm{t}^{30-32}$. Such devices consist simply of a three-dimensional configuration and a filter membrane with pores of specific shapes and sizes, which can be flexibly combined with other enrichment strategies ${ }^{33,34}$. Table 1 shows a comparison of the comprehensive performance of membranes based on size filtration over the past 10 years. Several research groups have reported that isolation by size of epithelial tumor cells (ISET) exhibits better performance than CellSearch in clinical tests of patients with various cancers ${ }^{35-37}$. Despite the feasibility of these size-based approaches in clinical applications, their disadvantage, which is that balancing the capture efficiency, purity, and cell viability, is usually difficult, cannot be ignored ${ }^{38}$. For these size-based approaches or devices, the key challenges are clogged pores and cell aggregation, which greatly reduce the selectivity of the CTC isolation process and affect the subsequent fluorescence imaging analysis ${ }^{10,39}$. The polycarbonate filter, which is inexpensive and user-friendly, was first used to enrich CTCs based on size, but randomly distributed or overlapping pores limit the capture efficiency, and the filter tends to become clogged ${ }^{32,36}$. With the development of microfabrication techniques, filters with different types and densities of pores could be precisely manufactured for the enrichment of $\mathrm{CTCs}^{31-34,37,38,40}$. Tang et al. ${ }^{38}$

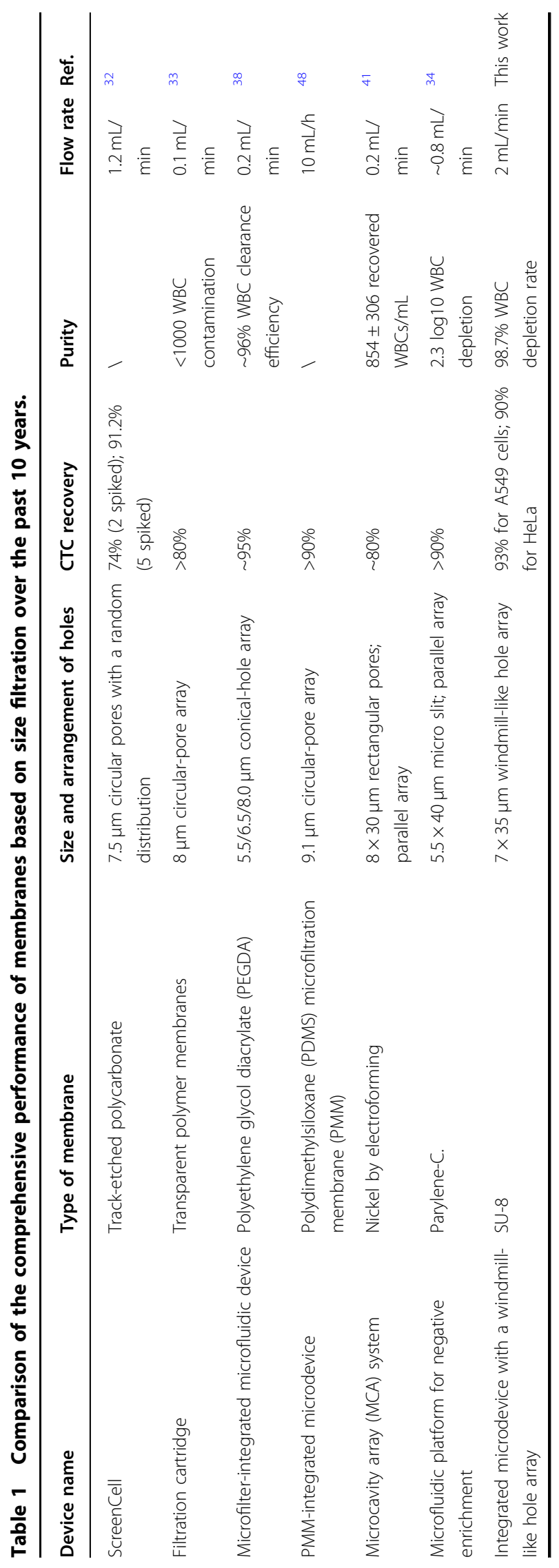


developed a microfluidic device with a microfilter of conical-shaped holes that obtained a higher WBC clearance efficiency ( 200 or $500 \mu \mathrm{L} / \mathrm{min}$ ) than that obtained with cylindrical-shaped holes because the conical shapes prevent the clogging of pores. Furthermore, Hosokawa et al. $^{41}$ reported that the number of WBCs remaining on the microcavity array (MCA) system is significantly reduced by changing the circular array to a rectangular array. Although optimizing the filter microstructure improves the WBC removal efficiency, hole clogging and cell aggregation on the micropores still occurs, which greatly hinders CTC capture and detection. Usually, the sample is diluted to a certain volume to prevent membrane clogging ${ }^{33,41}$. Furthermore, the flow rate, which directly affects the capture efficiency and the uniformity of cell dispersion, is also a key factor in this process ${ }^{33,38}$. As the cells accumulate on the filter membrane, the high flow rate inevitably increases the filtration pressure and applies it to each cell, which greatly increases the possibility that cells are crushed while passing through the filter. In general, a low flow rate ensures the desired effect of these membrane filtration-based approaches or devices, as shown in Table 1. Therefore, the rapid and efficient isolation of CTCs without clogging for membrane filtration still needs further exploration.

In this study, a novel integrated microdevice with a windmill-like hole array was developed for the efficient, clog-free and self-mixing enrichment of CTCs. The design of a windmill-like hole array on the SU-8 membrane enabled the cells to be dispersed more evenly and reduced the pressure on the cells. Furthermore, to improve the comprehensive performance of the SU-8 membrane, the surface was modified to avoid clogged pores and cell aggregation, which greatly reduced the number of cell clusters and improved the WBC clearance efficiency. Then, using the high-throughput detection and analysis device, the rapid and accurate identification of CTCs could be achieved. Under the optimal conditions, the advantages of the integrated microdevice for the rapid and efficient enrichment of CTCs without clogging were shown both theoretically and experimentally.

\section{Design and simulation}

Hole clogging and cell aggregation still exist, even though filter membranes with microholes of different sizes and shapes and in different arrangements have been microfabricated for CTC enrichment ${ }^{34,41-43}$. To resolve these problems, a windmill-like hole array was designed in this study to perturb the fluid above the microholes, which resulted in homogenization of the distribution of cells in the fluid and a reduced pressure on the cells (Fig. 1a). In nature, a windmill is driven to rotate by the passing airflow, and the airflow is in turn disturbed, which makes the airflow velocity uneven and disperses the pressure generated by the airflow. This phenomenon inspired a novel notion of applying the windmill disturbance model to CTC enrichment. Based on this idea, the microhole structure was first designed as a windmilllike hole array to achieve the clog-free and self-mixing enrichment of CTCs.

Theoretically, when fluid passes through a windmill-like hole array vertically, the fixed windmill-like hole array allows the fluid above the holes to self-mix because the forces are mutual. First, to verify the feasibility of this design, a $\mathrm{COMSOL}^{\circledR}$ simulation utilizing simplified boundary conditions and geometric models was performed to compare the fluid dynamics of three types of microhole designs $(7-\mu \mathrm{m}$-diameter circular hole array, $7 \times 35 \mu \mathrm{m}$ rectangular hole array and $7 \times 35 \mu \mathrm{m}$ windmilllike hole array). A scenario in which fluid flowed vertically at $200 \mu \mathrm{L} / \mathrm{min}$ through the filters $(80 \times 80 \mu \mathrm{m})$ with three different types of holes was simulated. Considering the mechanical strength and utilization of the SU-8 membrane, the center-to-center distance between neighboring holes was initially set to $30 \mu \mathrm{m}$. A Reynold's number of 1.0417 was obtained with the experimental parameters, which showed that the fluid flow in the chamber was generally laminar. To simplify our simulation, we assumed that the fluid was Newtonian and incompressible $^{44}$. The steady fluid flow in the mathematical model obeyed the Navier Stokes equations as follows:

$$
\begin{aligned}
& \rho(u \cdot \nabla) u=\nabla \cdot\left[-p I+\mu\left(\nabla u+(\nabla u)^{T}\right)\right]+F \\
& \rho \nabla \cdot u=0
\end{aligned}
$$

where $\rho$ and $\mu$ are the density and dynamic viscosity, respectively; $u$ and $p$ indicate the fluid velocity and fluid pressure, respectively; and $F$ is the external force that acts on the fluid.

Because the cells suspended in the fluid (or PBS solution) moved with the flow of the fluid, the status of fluidic flow could roughly reflect the status of cell movement. Figure $1 \mathrm{~b}-\mathrm{d}$ shows hydrodynamic simulations of steady fluid passing through holes. As shown in Fig. 1b, in the three-dimensional contour map of the fluidic velocity field, the fluidic flow within the blue area was relatively rapid $(1-6 \mathrm{~m} / \mathrm{s})$, whereas that outside the blue area was relatively stable (less than $1 \mathrm{~m} / \mathrm{s}$ ). The area where the fluidic flow was stable was prone to become a dead zone, where cells were easily deposited. The three-dimensional bule area above the windmill-like holes was significantly larger than that above the other two types of holes, which suggested that the cells moved more actively above the windmill-like hole array and had a lower possibility of being deposited on the surface of the dead zone at an equal distance from neighboring holes. Figure 1c shows the horizontal cross-sectional velocity field simulation at a height of $15 \mu \mathrm{m}$ above the holes. The fluidic velocity in 


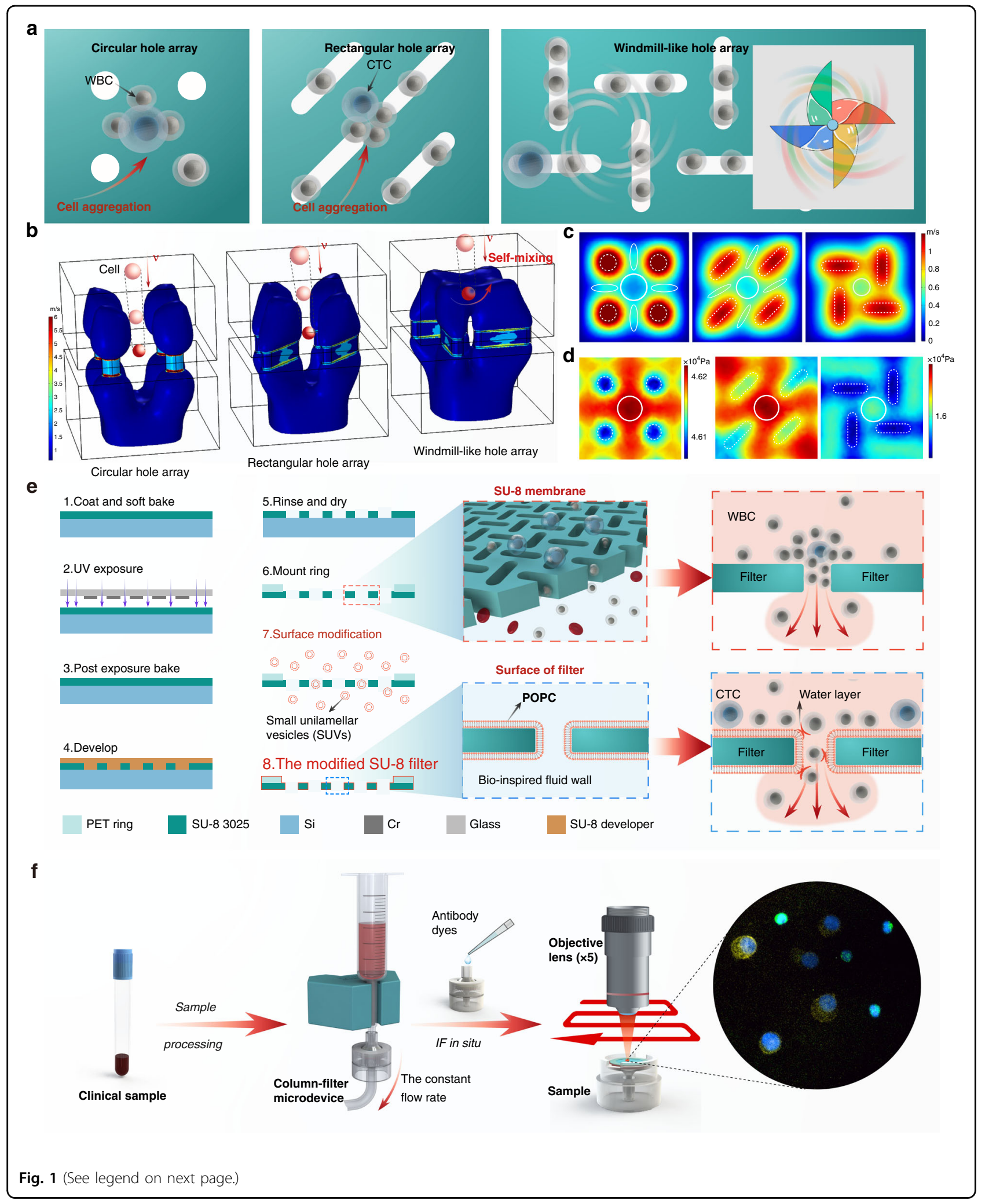


(see figure on previous page)

Fig. 1 Integrated microdevice with an SU-8 membrane containing a windmill-like hole array for the self-mixing enrichment of circulating tumor cells. a Schematic of the cell distribution on membranes with different types of pores. $\mathbf{b}-\mathbf{d}$ Hydrodynamic simulation of steady fluid passing through holes. $\mathbf{b}$ Three-dimensional contour map of the fluidic velocity field when the fluid passes through three different types of holes $(7 \mu \mathrm{m}-$ diameter circular hole array, $7 \times 35 \mu \mathrm{m}$ rectangular hole array and $7 \times 35 \mu \mathrm{m}$ windmill-like hole array). The fluidic flow within the blue area was relatively rapid (1-6 m/s), whereas the fluidic flow outside the blue area was relatively stable (less than $1 \mathrm{~m} / \mathrm{s})$. c Horizontal cross-sectional flow field simulation at a height of $15 \mu \mathrm{m}$ above the holes. $\mathbf{d}$ Horizontal cross-sectional pressure field simulation at a height of $15 \mu \mathrm{m}$ above the holes. The white circle is the dead area, where fluid velocity changed little due to the focusing effect of the fluid above the holes. The dotted frame represents the hole array. e Fabrication process of the SU-8 membrane with the windmill-like hole array. Using a special microfabrication technique, a homogeneous membrane with a windmill-like hole array could be fabricated accurately. Through surface modification, lipid bilayer-coated microholes on the SU-8 membrane were created to eliminate nonspecific adsorption between cells and the membrane. $\mathbf{f}$ Schematic of the experimental setup and operation flow. The processed blood sample passed through the separation column and the SU-8 membrane at a constant flow rate, labeled leukocytes were removed in the column, and CTCS were retained on the membrane. After the CTC enrichment process was completed, immunofluorescence was directly performed on the SU-8 membrane.

the dead area (the white circle) of the circular holes $(0.2-0.4 \mathrm{~m} / \mathrm{s})$ and parallel holes $(0.4-0.6 \mathrm{~m} / \mathrm{s})$ on the horizontal section was significantly lower than that found with the windmill-like holes $(\sim 0.8 \mathrm{~m} / \mathrm{s})$, which indicated that the circular hole array and rectangular hole array exerted a greater flow-focusing effect on the fluid, and cells were more likely to be deposited on the surface of the dead zone where fluidic flow was not active. Furthermore, to better understand the self-mixing process of the cells above the hole, the trajectory of the fluidic flow was simulated (Supplementary Fig. S1). A micro-vortex was formed above the windmill hole array with a smaller dead zone than the parallel hole array (Supplementary Fig. S1a, b). Due to the horizontal and vertical arrangements of the windmill-like hole array, the distribution of the fluid trajectory in the vertical section was asymmetrical, and the trajectories of cells above the area at an equal distance from neighboring vertical holes were more likely to change compared with that found for neighboring parallel holes before the cells reached the membrane surface. Even if the cell accidentally fell on the middle area of adjacent holes, it would slide into one of the holes because $\mathrm{F}_{\text {left }} \neq$ $\mathrm{F}_{\text {right }}$ (Supplementary Fig. S1c, d). In fact, the self-mixing process of the cells could be reflected by the changes in their trajectory. Therefore, the windmill-like hole array could exert a more obvious disturbing effect on the upper fluid, which would result in the self-mixing of cells.

Moreover, Fig. 1d shows that the partial pressure (the white circle) of the circular and rectangular hole arrays was markedly higher than $1.6 \times 10^{4} \mathrm{~Pa}$, whereas the partial pressure of the windmill-like hole array was slightly higher than $1.6 \times 10^{4} \mathrm{~Pa}$. For the same inlet flow rate, the pressure above the windmill-like hole array was significantly lower than that above the circular and rectangular hole array. In other words, under the same pressure conditions that the cells could withstand, the flow rate allowed by the windmill-like hole array was significantly higher than that allowed by the other two types of holes. Effectively, this novel design of a windmill-like hole array could homogenize the distribution of cells, prevent holes from clogging, and reduce the pressure on the membrane surface, which would allow a higher flow rate of fluid with cells passing through the holes. In the following sections, SU-8 membranes with different-sized holes based on a windmill-like hole array were fabricated for optimization, and the advantages of this design were further verified through comparisons with different membrane types.

\section{Results and discussion \\ Working principle of an integrated microdevice with a windmill-like hole array}

A scientific illustration of the developed SU-8 membrane with a windmill-like hole array is depicted in Fig. 1e, and the operation process using the membrane is systematically shown in Fig. 1f. After theoretically verifying the advantages of the windmill-like hole array, homogeneous membranes with pore sizes of $2 \times 10,3 \times 15,5 \times$ 25 , and $7 \times 35 \mu \mathrm{m}$ were fabricated accurately using a series of microfabrication techniques (Fig. 2a). Moreover, membranes with the windmill-like hole array could be easily integrated into the syringe filter holder (Supplementary Fig. S2). The processed blood sample passed through the column-filter microdevice at a constant flow rate, labeled leukocytes removed in the column, and CTCs were retained on the membrane. After the CTC enrichment process was completed, immunofluorescence (IF) was directly performed on the membrane. The simple operation process could meet the clinical requirements.

\section{Surface modification of the SU-8 membrane to reduce nonspecific cell adsorption}

Our preliminary experiments showed the formation of cell clusters that caused pore blockages due to the nonspecific adsorption of materials to cells (Fig. 2b). The materials were not specially treated to reduce the nonspecific adsorption. Eventually, the recovery efficiency of CTCs would decrease due to the increased pressure applied to each cell, and it would be difficult for our 


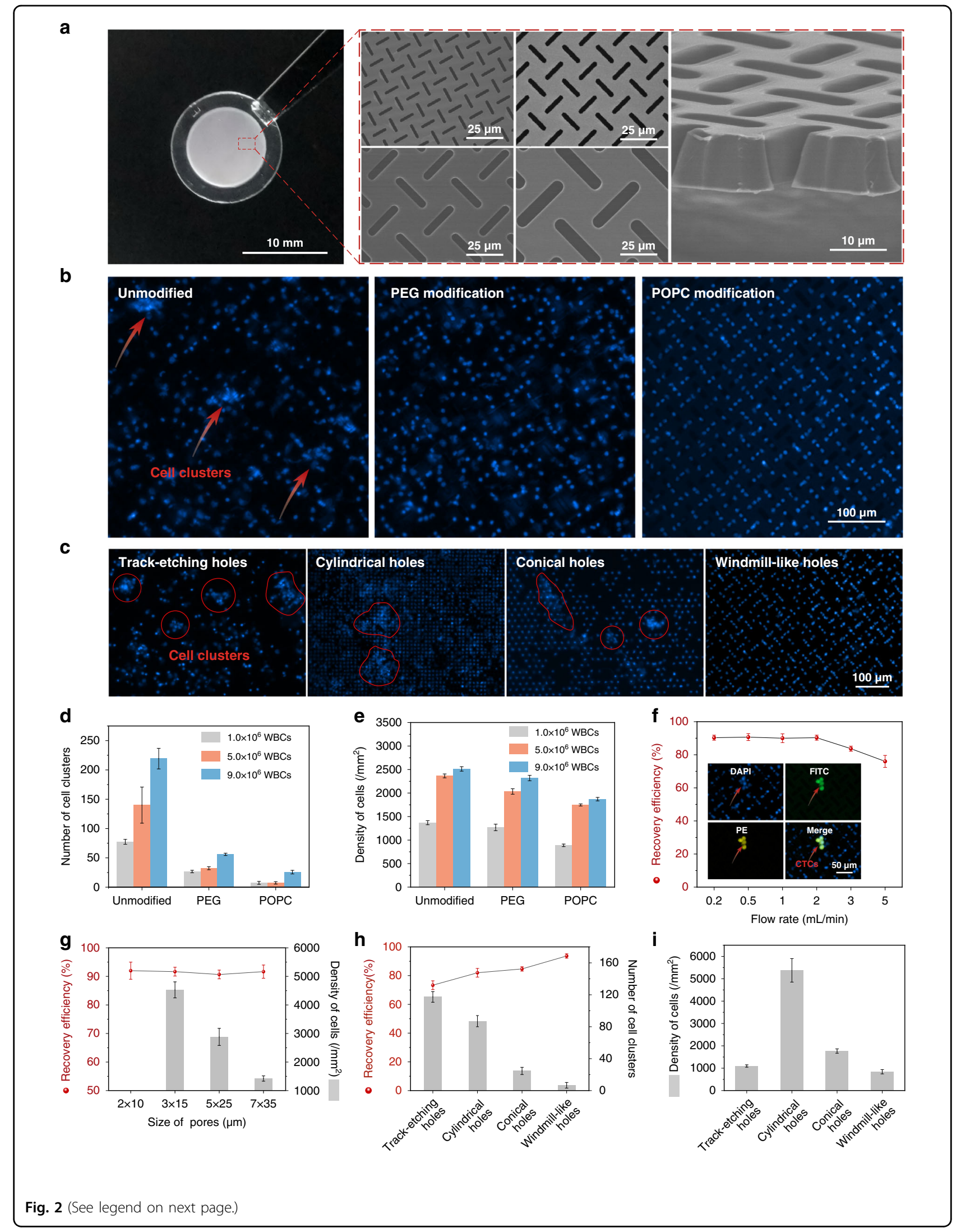




\begin{abstract}
(see figure on previous page)
Fig. 2 Optimization of the SU-8 membrane and filtering conditions to improve the comprehensive performance of platform. a SEM images and physical photograph of the SU-8 membrane. SU-8 membranes with a diameter of $13 \mathrm{~mm}$, an effective diameter of $9 \mathrm{~mm}$ and a thickness of $10 \mu \mathrm{m}$ were obtained with four hole sizes: $2 \times 10 \mu \mathrm{m}, 3 \times 15 \mu \mathrm{m}, 5 \times 25 \mu \mathrm{m}$ and $7 \times 35 \mu \mathrm{m}$. $\mathbf{b}$ Effect of different surface modification conditions on the cell distribution. WBC $\left(1.0 \times 10^{6}\right)$ were spiked in $10 \mathrm{~mL}$ of PBS and filtered at $2 \mathrm{~mL} / \mathrm{min}$ under different conditions (unmodified, PEG and POPC). The red arrows indicate cell clusters. $\mathbf{c}$ Effect of different types of holes on the cell distribution. WBCs $\left(1.0 \times 10^{6}\right)$ were spiked in $10 \mathrm{~mL}$ of PBS and filtered at $2 \mathrm{~mL} / \mathrm{min}$ using four types of holes (PC with $8 \mu \mathrm{m}$-diameter track-etching holes, SU-8 with $7 \mu \mathrm{m}$-diameter cylindrical holes, PEGDA with 8 - $\mu \mathrm{m}$ conical holes and SU-8 with $7 \times 35 \mu \mathrm{m}$ windmill-like holes). The red circles and arrows indicate cell clusters. $\mathbf{d}$, e Effect of different surface modification conditions on the number of cell clusters and the cell density. WBCs $\left(1.0 \times 10^{6}, 5.0 \times 10^{6}\right.$, and $\left.9.0 \times 10^{6}\right)$ were spiked in $10 \mathrm{~mL}$ of PBS and filtered at $2 \mathrm{~mL} / \mathrm{min}$ under different conditions using SU-8 with $7 \times 35 \mu \mathrm{m}$ windmill-like holes. f, g Optimization of conditions for CTC enrichment. The sample contained $4 \times 10^{6} \mathrm{WBCs}$ and approximately 400 labeled CTCS. CTCs were accurately identified as DAPI ${ }^{+}, \mathrm{FICC}^{+}$, and PE ${ }^{+}$. $\mathbf{h}, \mathbf{i}$ Performance comparison among different types of holes under optimal conditions. The sample containing $4 \times 10^{6}$ WBCs and $\sim 400$ labeled CTCs was filtered at $2 \mathrm{~mL} / \mathrm{min}$. The error bars correspond to the standard deviation from three independent experiments.
\end{abstract}

instrument to identify and analyze all the cells accurately based on cell clusters. To improve the performance of the windmill-like array holes on the SU-8 membrane and meet the requirements of the instrument to accurately identify cells, reducing the nonspecific adsorption between materials and cells was vital. Previous studies have demonstrated that hydrophilicity ${ }^{45}$ including lipid coating $^{46}$ and polyethylene glycol (PEG) modification ${ }^{47}$ could eliminate the nonspecific binding between proteins and material surfaces. Based on previous work, we chose hydrophilic modifications such as 1-palmitoyl-2-oleoylsn-glycero-3-phosphocholine (POPC) and PEG to improve the results.

To assess the effect of different modification types on the $7 \times 35 \mu \mathrm{m}$ SU- 8 membrane, the number of cell clusters and the cell density under three conditions (unmodified, PEG and POPC) were evaluated. The cell suspension containing different numbers of WBCs was thoroughly mixed to prevent errors caused by adhesion and then added to the device at a flow rate of $2 \mathrm{~mL} / \mathrm{min}$. After 4',6-diamidino-2-phenylindole (DAPI) staining of the filter membrane, the cell distribution was qualitatively observed, and the number of cell clusters and cell density were then quantitatively determined using the highthroughput CTC detection device. Unsurprisingly, remarkable differences in cell uniformity could be observed after the different treatments of the SU-8 membrane (Fig. 2b). Under unmodified conditions, the holes in the filter membrane were prone to blockage and induced the formation of cell clusters that were not conducive to the identification of the instrument. In contrast, the POPC and PEG modifications reduced the occurrence of blockages and made cells uniform according to the hole distribution, which made instrument identification and analysis easy.

Furthermore, we conducted a quantitative analysis of the various modification effects, as shown in Fig. 2d, e. Compared with the effect of the unmodified conditions, both the POPC and PEG modifications reduced the nonspecific adsorption of the filter to a certain extent, but the effect of the POPC modification was significantly better than that of the PEG modification. Specifically, for $1.0 \times 10^{6}, 5.0 \times 10^{6}$, and $9.0 \times 10^{6} \mathrm{WBCs}$, the number of clusters under the POPC (PEG) modification was reduced by $90.9 \%$ (66.2\%), $95.0 \%$ (77.1\%), and $88.1 \%$ (74.4\%), respectively, compared with that found under unmodified conditions. Moreover, for the same number of WBCs, the lowest cell density was found under the POPC modification. When analyzing the cell density, the instrument excluded the cell clusters formed by multiple cells; therefore, the actual value of the cell density on the unmodified filter membrane was higher than the measured value, but this finding did not affect our comparison of the modification effects. In short, the POPC modification was more conducive to reducing clogging and background interference in the filter membrane.

\section{Optimization of the conditions for CTC enrichment}

After the optimal conditions for WBC depletion that yielded the desired effect were explored (Supplementary Fig. S3), we further optimized the filtration conditions for CTC capture. In the mechanical capture of CTCs, the filtration conditions including the dilution volume, flow rate and filter pore size had certain impacts on the recovery and purity of $\mathrm{CTCs}^{33}$. Meunier et al. ${ }^{33}$ proved that the efficiency reached the upper limit with a PBS: blood ratio of 6:1, and dilution was not an effective means to reduce background interference. Therefore, the dilution volume was initially set to $10 \mathrm{~mL}(>6 \mathrm{~mL})$ to ensure a low transmembrane pressure and a high recovery rate, and the flow rate and filter pore size were optimized to achieve the desired effect. For all optimization experiments, the sample contained $4 \times 10^{6} \mathrm{WBCs}$ and $\sim 400$ CTCs. In the range of flow rates considered $(0.2-5 \mathrm{~mL} /$ min with membranes consisting of a $7 \times 35 \mu \mathrm{m}$ hole array), we observed a decreased recovery efficiency when the flow rate was higher than $2 \mathrm{~mL} / \mathrm{min}$ (Fig. 2f). This finding was obtained because in our system, a flow rate 
that was too high eluted the cells that magnetically adsorbed on the spheres in the column, which increased the number of cells and the transmembrane pressure on the filter. Finally, filtering at $2 \mathrm{~mL} / \mathrm{min}$ was identified as the optimal condition for achieving high efficiency and adopted for subsequent experiments. Compared with the other membrane flow rates ${ }^{33,38}$, a higher flow rate $(2 \mathrm{~mL} /$ min) was needed to achieve the same effect with our system. This finding was obtained because the microstructure on the membrane greatly reduced the transmembrane pressure on the cells, and as a result, the membrane would withstand a greater flow rate without any loss of CTCs (Fig. 1d).

The pore size is expected to be a key factor in the efficiency and purity of CTC enrichment. Hosokawa et al. reported that a rectangular MCA with a size of $8 \mu \mathrm{m}$ achieved the best balance between high purity and high recovery ${ }^{41}$. Based on this fact, our membrane with a size less than $8 \mu \mathrm{m}$ was selected for obtaining a higher recovery rate. Evidently, the cell density on the membrane would increase as the size of the rectangular pores decreased. The depletion of upstream WBCs and the high porosity $(30-50 \%)$ of the SU-8 membrane could reduce the phenomenon that the background interference increased as rectangular pore size decreased. In addition, even if there were many cells on the membrane, all cells, including a few CTCs, were accurately identified by the high-throughput CTC detection device as long as the cells were evenly distributed on the filter membrane without aggregation. As shown in Fig. 2a, we fabricated SU-8 membranes with pore sizes of $2 \times 10,3 \times 15,5 \times 25$, and $7 \times 35 \mu \mathrm{m}$ for tests. Supplementary Fig. S4 qualitatively shows that the number of cells gradually decreased as the pore size increased. Among the membranes, the membrane with $2 \times 10 \mu \mathrm{m}$ pore sizes had the most cells and the most serious aggregation, which could not be recognized by the instrument (Supplementary Fig. S4a). Figure $2 \mathrm{~g}$ quantitatively shows the specific recovery rate and cell density obtained with each pore size. With a pore width of less than $8 \mu \mathrm{m}$, all recovery rates were higher than 90\%; however, the membrane with a pore size of $7 \times 35 \mu \mathrm{m}$ exhibited the lowest background interference, and the cell density was $1423.0 \pm 92.7$. Therefore, the SU-8 membrane with a pore size of $7 \times 35 \mu \mathrm{m}$ was used as the optimal material for subsequent experiments.

\section{Performance comparison with the traditional membrane}

To further assess the practicability of the SU-8 membrane with a windmill-like hole array, $\sim 400$ CTCs spiked in a $1 \mathrm{~mL}$ cell suspension containing $10^{6}$ WBCs were used to evaluate the comprehensive performance of the different types of membranes (PC with $8 \mu \mathrm{m}$ track-etching holes, SU-8 with $7 \mu \mathrm{m}$ cylindrical holes, PEGDA with $8 \mu \mathrm{m}$ conical holes, and SU-8 with $7 \times 35 \mu \mathrm{m}$ windmill- like holes). CTCs were accurately identified by DAPI ${ }^{+}$, FITC $^{+}$, and $\mathrm{PE}^{+}$(Supplementary Fig. S5). By the DAPI staining of nucleated cells, the distribution of cells on different types of membranes was observed, which suggested that the windmill-like hole array on SU-8 could effectively prevent cell aggregation (Fig. 2c).

Furthermore, the recovery rate of CTCs and the number of cell clusters were counted using different types of membranes. As shown in Fig. $2 \mathrm{~h}$, compared with other membranes at a flow rate of $2 \mathrm{~mL} / \mathrm{min}, \mathrm{SU}-8$ with a $7 \times$ $35 \mu \mathrm{m}$ windmill-like hole array yielded the highest recovery rate $(93.7 \pm 1.5 \%)$ and the lowest number of cell clusters $(6.7 \pm 3.5)$. In addition, the cell density on the SU8 membrane with the windmill-like hole array was the lowest $(842.3 \pm 96.0)$, which indicated that the windmilllike hole array could eliminate WBCs with the greatest efficiency (Fig. 2i). In short, the SU-8 membrane with the windmill-like hole array showed advantages for comprehensive performance compared with traditional membranes, which means that subsequent experiments could be conducted based on this finding.

\section{Validation using simulated clinical samples}

To verify the potential clinical application value of the SU-8 membrane, the recovery efficiency, the WBC depletion rate and the detection limit of the integrated microdevice were comprehensively evaluated under the optimal conditions previously explored. The samples in this set of experiments contained 0-100 live CTCs (A549 and HeLa) and more complex blood from the hospital, which was close to that from cancer patients in the clinic. After sample processing and CTC enrichment, the samples were subjected to IF in situ and identified using a high-throughput detection device (Fig. 1f). Figure 3a-c shows the distinguishing of tumor cells from other cells. Cells that were $\mathrm{DAPI}^{+}, \mathrm{CD}_{4} 5^{+}$, and $\mathrm{EpCAM}^{-}$were considered WBCs (Fig. 3a). A549 and HeLa cells were identified as $\mathrm{DAPI}^{+}, \mathrm{CD}^{3} 5^{-}$, and $\mathrm{EpCAM}^{+}$cells (Fig. 3b, c). In addition, during the experiments, a few doublepositive cells $\left(\mathrm{CD} 45^{+}\right.$and $\left.\mathrm{EpCAM}^{+}\right)$and double-negative cells $\left(\mathrm{CD} 45^{-}\right.$and $\left.\mathrm{EpCAM}^{-}\right)$were observed (Fig. 3d, e). We speculated that the double-positive cells might be caused by the autofluorescence of the cells due to drying of the sample, and the double-negative cells might be caused by low expression or no expression of EpCAM on CTCs. The cells that we could not judge were all excluded from the CTC enumeration.

It was more challenging to obtain a high recovery efficiency when CTCs were spiked into blood at a low concentration $(<100$ cells per milliliter of blood) compared with that obtained with a high CTC concentration (1000 cells per milliliter of blood ${ }^{41}$. When the CTC concentration was low, the loss of one tumor cell would also have a great impact on the recovery efficiency. Therefore, 


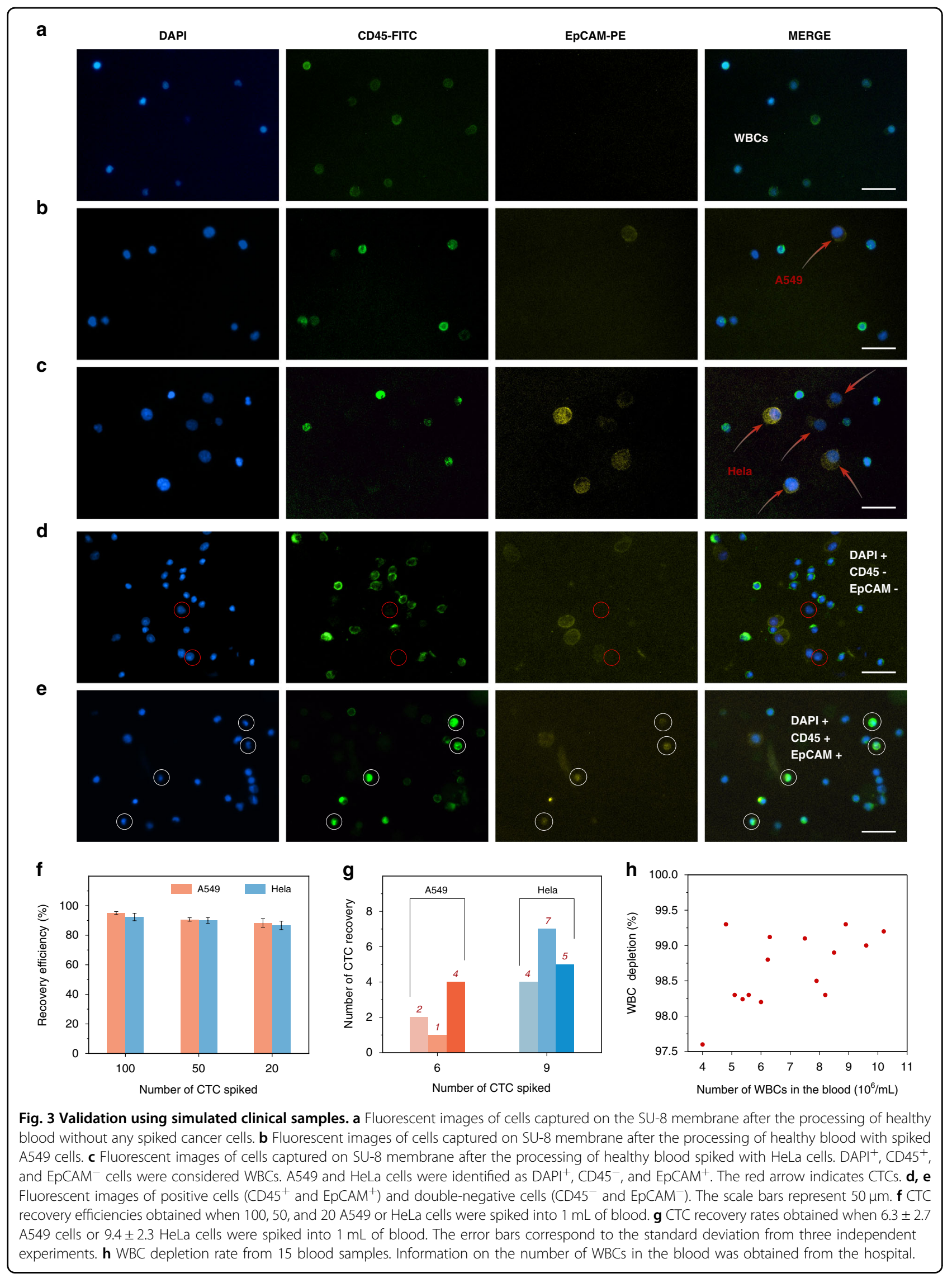


there was a more stringent requirement for the equipment and methods to capture CTCs from samples with a low concentration of CTCs. Regardless of this fact, recovery rates of $95.0 \pm 1 \%, 90.7 \pm 1.2 \%$, and $88.3 \pm 2.9 \%$ were achieved with the spiking of 100, 50, and 20 A549 cells, respectively; and recovery rates of $92.3 \pm 2.5 \%$, $90.0 \pm 2.0 \%$, and $86.7 \pm 2.9 \%$ were achieved with the spiking of 100, 50, and 20 HeLa cells, respectively (Fig. 3f). Recovery rates of $93 \%$ and $90 \%$ were obtained for A549 and HeLa cells, respectively, with a CTC concentration of 20-100 cells per milliliter of blood. Clearly, the fewer the number of cells spiked, the greater the deviation this spiking caused to the results. It was difficult for us to guarantee the actual number when the number of cells spiked was less than 10 . The average and standard deviation were calculated to reduce the deviation of the cells spiked from a statistical point of view after counting the number of CTCs contained in $10 \mu \mathrm{L}$ of the cell suspension five times. As shown in Fig. 3g, we performed three repeated experiments with A549 and HeLa cells. When $6.3 \pm 2.7$ A549 cells were spiked, we captured 2, 1, and 4 cells; and when $9.4 \pm 2.3 \mathrm{HeLa}$ cells were spiked, we captured 4, 7, and 5 cells. In addition, the WBC depletion rate from 15 experiments was recorded, and the average was $98.7 \%$ (Fig. 3h). As expected, our platform still obtained a $93 \%$ recovery rate for A549 cells and a $90 \%$ recovery rate for HeLa cells when the CTC concentration was $20-100$ cells per milliliter of blood, the detection limit was less than 10 cells per milliliter of blood, and the WBC depletion rate was $98.7 \%$. By comparing the indicators of our device with the same indicators of other devices in the past decade, our device could not only guarantee a high recovery rate and high purity but also greatly improve the sample processing speed (Table 1). In addition, other advantages, such as reducing clogging and homogenizing the cell distribution, are not quantitatively shown in the table. Considering the desired effect achieved by our device, real clinical samples could be used for a more comprehensive evaluation.

\section{Clinical test}

With ethical approval from the cooperative hospital (Validation date of 2021/08/27, Report No. IRB2021055), blood samples from cancer patients were obtained for clinical trials. A total of 20 blood samples donated by 5 healthy donors and 15 patients with different types of cancer were tested using our integrated microdevice with a windmill-like hole array. The volume of each blood sample was $1-3 \mathrm{~mL}$. The pretreated blood sample was loaded into the column-filter microdevice containing $10 \mathrm{~mL}$ of PBS for full dilution before filtration. The filtration experiments were carried out using the SU-8 membrane with a $7 \times 35 \mu \mathrm{m}$ windmill-like hole array at a flow rate of $2 \mathrm{~mL} / \mathrm{min}$. In addition, the SU-8 membrane was previously processed by POPC. After filtration and immunofluorescence in situ, fluorescence images of the cells on the membranes were obtained using the CTC high-throughput detection device (blue represents DAPI staining of all the cells, the green color shows $\mathrm{CD} 45^{+}$, and the yellow color indicates $\mathrm{EpCAM}^{+}$).

As shown in Fig. 4, four examples of CTC fluorescence images captured using the blood from different cancer patients are listed. More fluorescence images of CTCs are shown in Supplementary Fig. S6. EpCAM ${ }^{+}$and CD $45^{-}$ cells (CTCs) were captured using blood samples from multiple cancer patients, verifying the feasibility of using our platform for CTC isolation and detection. Table 2 shows the test results from all blood samples, including healthy donors and cancer patients. CTCs could hardly be detected in the blood samples from healthy donors, indicating that our device exhibits a low number of falsepositives. Statistically, CTCs were identified in 11 of 15 cancer patients (73.3\% detection rate); however, no CTC signature was found in the blood of some cancer patients. Two reasons might explain these findings. On the one hand, the epithelial markers such as EpCAM on the CTCs of these cancer patients might be downregulated or lost due to EMT. On the other hand, there might be no CTCs in the sample based on the limited clinical sample volume. In addition, the cells on the membrane were evenly distributed along with the arrangement of the holes, and the particularly obvious phenomenon of cell aggregation and clogged pores was not observed, which greatly improved the accuracy and convenience of detecting captured CTCs using our device (Supplementary Fig. S7). Of course, double-negative cells (CD45 and $\mathrm{EpCAM}^{-}$) were still observed on the membrane, but their appearance did not affect our statistical results. Furthermore, further clinical trials should be conducted with more detailed analyses to evaluate whether an integrated microdevice with a windmill-like hole array is a more appropriate tool for the detection of CTCs from metastatic tumors in patients.

\section{Conclusion}

In conclusion, an integrated microdevice with an SU-8 membrane containing a windmill-like hole array was developed for the enrichment of CTCs. First, the advantages of the windmill-like hole array design were validated theoretically through comparisons of hydrodynamic simulations with different microhole designs. To improve the comprehensive performance of the windmill-like hole array, different surface modifications were conducted; the POPC modification, which reduced the number of cell clusters by $91.3 \%$ and the cell density by $29.0 \%$ on average, was considered the best solution. Using a leukocyte suspension spiked with labeled tumor cells, the optimal conditions (flow rate of $2 \mathrm{~mL} / \mathrm{min}$ and pore size of 


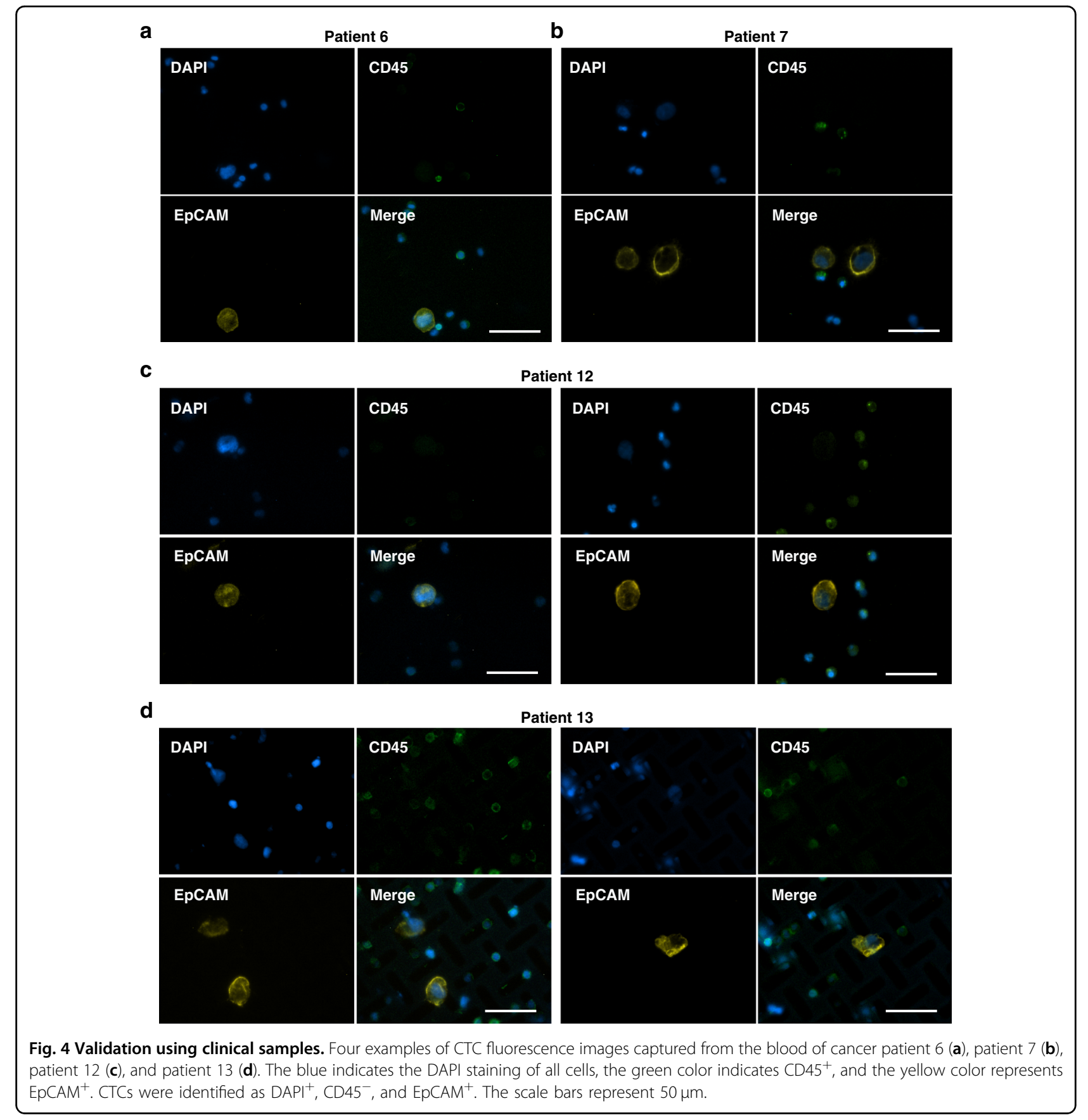

$7 \times 35 \mu \mathrm{m})$ for CTC enrichment were applied to obtain the desired effect. Furthermore, it was experimentally demonstrated that the SU-8 membrane with the windmill-like hole array could induce the self-mixing of fluid to prevent pores from becoming clogged and reduce the possibility of cell aggregation compared with that achieved with traditional membranes. Moreover, the clinical practicability of this device was estimated under the optimal conditions using healthy blood samples with
0-100 live tumor cells, and the device achieved a recovery efficiency of $93 \%$ for A549 cells, a recovery efficiency of 90\% for HeLa cells, a WBC depletion rate of $98.7 \%$ and a detection limit of less than 10 cells per milliliter of blood. The advantages of the integrated microdevice for the rapid and efficient enrichment of CTCs without clogging were shown both theoretically and experimentally. Therefore, an integrated microdevice with an SU-8 membrane consisting of a windmill-like hole array has 
Table 2 Validation using clinical samples.

\begin{tabular}{|c|c|c|c|}
\hline $\begin{array}{l}\text { No. of } \\
\text { patient }\end{array}$ & Type of cancer & $\begin{array}{l}\text { Volume of } \\
\text { blood }(\mathrm{mL})\end{array}$ & $\begin{array}{l}\text { Number } \\
\text { of CTCs }\end{array}$ \\
\hline 1 & Healthy donor & 2 & 0 \\
\hline 2 & & 2 & 0 \\
\hline 3 & & 2 & 0 \\
\hline 4 & & 2 & 0 \\
\hline 5 & & 2 & 0 \\
\hline 6 & Liver cancer & 1 & 1 \\
\hline 7 & & 1.5 & 2 \\
\hline 8 & & 2.5 & 4 \\
\hline 9 & & 1.5 & 3 \\
\hline 10 & & 2 & 0 \\
\hline 11 & Lung cancer & 2 & 0 \\
\hline 12 & & 1.2 & 2 \\
\hline 13 & & 1.2 & 3 \\
\hline 14 & Breast cancer & 3 & 1 \\
\hline 15 & & 2.5 & 2 \\
\hline 16 & Stomach cancer & 1 & 4 \\
\hline 17 & & 1 & 2 \\
\hline 18 & Bladder cancer & 1 & 0 \\
\hline 19 & Colon cancer & 1 & 0 \\
\hline 20 & Bile duct tumor & 1 & 1 \\
\hline
\end{tabular}

the potential to be a tool for the clinical application and more detailed analyses of CTCs.

\section{Materials and methods}

Materials and sample preparation

Blood samples from healthy individuals and cancer patients were collected in EDTA-treated tubes at the Affiliated Suzhou Science and Technology Town Hospital, Nanjing Medical University. Isopore ${ }^{\mathrm{TM}}$ polycarbonate membranes were purchased from Merck Millipore. PEGDA membranes with $8 \mu \mathrm{m}$ conical holes were purchased from An Fang Bio (Guangzhou, China). SU-8 membranes with different sizes and syringe filter holders were processed by TopMembranes Technology Company (Shenzhen, China). PBS, fetal bovine serum (FBS), penicillin/streptomycin, RPMI medium 1640, DMEM, 0.25\% trypsin-EDTA, 1-step fix/lyse solution (10x), fluorescent molecule labeled or biotin-conjugated antibody, and dimethyl sulfoxide (DMSO) were purchased from Thermo Fisher. Streptavidin-functionalized magnetic beads $(2.8 \mu \mathrm{m})$ were obtained from BioMag beads (Wuxi, China). A549 and HeLa cells were purchased from Cobioer Biosciences Company (Nanjing, China).
Mesophilic-2000 (PEG) was purchased from Meso Biosystems Company (Wuhan, China), and POPC was purchased from Aladdin.

\section{SU-8 membrane fabrication and surface modification}

Figure 1e shows the fabrication and surface modification process of the SU-8 membrane with a windmill-like hole array. First, the silicon substrate was spin coated with a 10- $\mu \mathrm{m}$-thick negative resist (SU-8 3025) and soft baked at $95^{\circ} \mathrm{C}$ for $10 \mathrm{~min}$. A chromium mask was then used to pattern the holes under UV light $\left(180 \mathrm{~J} / \mathrm{cm}^{2}\right)$, and a postexposure bake occurred directly after exposure. After development, rinsing, drying and mounting of the PET ring, SU-8 organic membranes with a diameter of $13 \mathrm{~mm}$, an effective diameter of $9 \mathrm{~mm}$ and a thickness of $10 \mu \mathrm{m}$ were obtained. Approximately 250,000 microslits (2-7 $\mu \mathrm{m}$ in width and $10-35 \mu \mathrm{m}$ in length) were arranged vertically and periodically (Fig. 2a). The standard deviation (SD) of the pore diameter was less than $3 \%$, and the porosity was up to $50 \%$. Finally, the SU-8 membrane was soaked in SUVs (POPC) $)^{46}$ or PEG solution for surface modification.

\section{Experimental setup}

The experimental setup (column-filter microdevice) was composed of four parts: a separation column, a syringe filter holder, an SU-8 membrane and a peristaltic pump (IPC; Ismatec, Wertheim, Germany). Supplementary Fig. S2 shows the specific structure of the syringe filter holder. The processed blood sample passed through the separation column and the SU-8 membrane at a constant flow rate provided by the peristaltic pump, labeled leukocytes were removed from the column, and CTCs were retained on the membrane. After the negative enrichment process was completed, the subsequent procedures, including washing, blocking, and immunofluorescence, were directly performed for the SU-8 membrane. Figure $1 \mathrm{f}$ shows a schematic representation of the experimental setup and operating flow.

\section{Cell culture and cell spiking}

A549 cells were cultured in RPMI 1640 containing 10\% FBS and $1 \%$ penicillin/streptomycin. HeLa cells were cultured in basic DMEM supplemented with $10 \%$ FBS and $1 \%$ penicillin/streptomycin. All cell lines were grown and maintained at $37{ }^{\circ} \mathrm{C}$ with $5 \% \mathrm{CO}_{2}$ in a humidifiedatmosphere. The cells were harvested with $0.25 \%$ trypsinEDTA and resuspended in PBS before use.

In the preliminary optimization experiment, only 400 labeled CTCs were mixed with a WBC suspension from blood to optimize the parameters. To simulate the actual situation of clinical cancer patients and verify the practicability of the platform, $10 \mu \mathrm{L}$ of a suspension of A549 or HeLa cells $(10,20,50$, or 100 CTCs per $10 \mu \mathrm{L})$ was spiked into $1 \mathrm{~mL}$ of blood for testing under the 
optimal conditions. In addition, the exact number of cells spiked into the blood was controlled by counting them multiple times on a somatic cell counting slide (Citotest; Suzhou, China).

\section{CTC isolation}

Clinical samples or simulated samples (blood mixed with CTCs) needed to be preprocessed before CTC isolation. One milliliter of the blood sample was lysed and fixed for $15 \mathrm{~min}$ with $10 \mathrm{~mL}$ of one-step fix/lyse solution $(1 \times)$ and then centrifuged to retain the cell pellet. The sample was diluted with $200 \mu \mathrm{L}$ of PBS, specifically labeled with $2.5 \mu \mathrm{L}$ of anti-human CD45 biotin and incubated for $20 \mathrm{~min}$. The sample was then incubated for 15 min with $2.5-20 \mu \mathrm{L}$ of streptavidin- magnetic beads $(10 \mathrm{mg} / \mathrm{mL})$. Before sample introduction, the columnfilter device was rinsed and filled with PBS. The processed sample was then added to the device and mixed well with PBS. The diluted sample was driven to pass through the column-filter device at a stable flow rate by the negative pressure generated by the peristaltic pump. In addition, the flow rate could be controlled by changing the peristaltic pump's speed. When the sample was reduced to the filling height of the steel ball, PBS was introduced to rinse the device.

\section{Cell immunostaining}

After microfiltration of the sample solution, the separation column was removed, and the captured cells on the SU-8 membrane were treated inside the syringe filter holder. Two hundred microliters of $0.2 \%$ Triton $\mathrm{X}-100$ in PBS was introduced to the filter holder and incubated for $5 \mathrm{~min}$ to permeabilize cells, and $200 \mu \mathrm{L}$ of PBS was then introduced at $50 \mu \mathrm{L} / \mathrm{min}$ to rinse the sample. Similarly, the sample on the membrane was incubated for 5 min with $200 \mu \mathrm{L}$ of $1 \%$ BSA in PBS to block nonspecific binding and rinsed with $200 \mu \mathrm{L}$ of PBS at $50 \mu \mathrm{L} /$ min. The cells on the membrane were then incubated with $200 \mu \mathrm{L}$ of immunostaining solution containing antiEpCAM PE (1:40 dilution) and anti-human CD45 FITC (1:80 dilution) at $4{ }^{\circ} \mathrm{C}$ in the dark for $30 \mathrm{~min}$ and rinsed with $200 \mu \mathrm{L}$ of PBS at $50 \mu \mathrm{L} / \mathrm{min}$. After staining with $1 \mu \mathrm{g} /$ $\mathrm{mL}$ DAPI for $8 \mathrm{~min}$, the sample was rinsed with $200 \mu \mathrm{L}$ of PBS at $50 \mu \mathrm{L} / \mathrm{min}$. The screw cap, gland, and silicone gasket were then carefully removed from the syringe filter holder, and 10-20 $\mu \mathrm{L}$ of ProLongTM Diamond Antifade Mountant with DAPI (Thermo Fisher) was dropped on the membrane. Finally, a cover glass was carefully mounted to avoid air bubbles.

\section{Cell identification and statistical analysis}

The sample was placed into the injection port of the high-throughput detection and analysis device that we previously built. The automatic stage moved in the direction set by the program to scan through the entire membrane, which yielded DAPI, FITC, and PE fluorescence images. The Spinnaker SDK software that we wrote was used to stitch all the acquired cell images and complete cell counting and analysis. To ensure the accuracy of CTC counting, experienced cell biologists reanalyzed all fluorescence images artificially, and counted the number of CTCs after analyzing them with the software. In addition, the CTC recovery, cell density, and WBC depletion were obtained as follows:

$$
\begin{aligned}
& \text { Recovery efficiency }(\%)=\frac{N_{R}}{N_{S}} \\
& \text { Density of cells }=\frac{C_{R}}{S_{E}} \\
& \text { WBC depletion }(\%)=\frac{C_{S}-C_{R}}{C_{S}}
\end{aligned}
$$

where $N_{R}$ and $N_{S}$ are the number of CTCs captured on the membrane and the total number of CTCs, respectively, spiked into the leukocyte suspension or blood, respectively; $C_{R}$ and $C_{S}$ are the number of cells retained on the membrane and the total number of cells spiked, respectively; $S_{E}$ is the effective area of the filter membrane; and $N_{R}$ and $C_{R}$ were directly counted by a highthroughput detection and analysis device.

\section{Acknowledgements \\ This work was supported by the National Key R\&D Program of China (No. 2021YFC2500401), the National Natural Science Foundation of China (No. 61874133, No. 61901469, No. 22005331), the Key Research and Development Program of Jiangsu Province (No. BE2018080, No. BE2019684, No. BE2020768), the Jihua Laboratory Foundation (No. X190181TD190), the Youth Innovation Promotion Association of CAS (No. 2019322, No.2018360, No. Y201856), the Instrument Developing Project of the Chinese Academy of Sciences (No. YJKYYQ20190057, No. YJKYYQ20200046, No. ZDKYYQ20210004), and the Science and Technology Development Program of Suzhou (No. SYG201907). We acknowledge the patients, donors, and nurses that participated in the blood donation and collection and the technical support provided by TopMembranes Technology Company.}

\footnotetext{
Author details

${ }^{1}$ School of Biomedical Engineering (Suzhou), Division of Life Sciences and Medicine, University of Science and Technology of China, 260026 Hefei, China. ${ }^{2}$ CAS Key Lab of Bio-Medical Diagnostics, Suzhou Institute of Biomedical Engineering and Technology, Chinese Academy of Sciences, 215163 Suzhou, China. ${ }^{3} \mathrm{Ji}$ Hua Laboratory, 528000 Foshan, China. ${ }^{4}$ Department of Laboratory Medicine, The Affiliated Suzhou Science and Technology Town Hospital, Nanjing Medical University, 215153 Suzhou, China. ${ }^{5}$ Shenzhen Institute for Advanced Study, University of Electronic Science and Technology of China, 518000 Shenzhen, China. ${ }^{6}$ Suzhou CASENS Co., Ltd, 215163 Suzhou, China. ${ }^{7}$ Jinan Guoke Medical Technology Development Co., Ltd, 250001 Jinan, China

\section{Author contributions}

H.L.: Conceptualization, Methodology, Data curation, Writing -original draft, Visualization, Writing —review and editing. J.L.: Resources. Z.Z.: Software, Validation. Z.G.: Resources. C.Z.: Resources. Z.W.: Validation. Q.G.: Resources. C.L.: Resources. C.L.: Resources. J.Y.: Resources. A.Z.: Resources. J.X.: Resources. Q.G.: Validation. W.Z.: Conceptualization, Methodology, Writing-review and editing. L.Z.: Conceptualization, Methodology, Writing-review and editing.
} 


\section{Conflict of interest}

The authors declare no competing interests.

Supplementary information The online version contains supplementary material available at https://doi.org/10.1038/s41378-021-00346-y.

Received: 29 July 2021 Revised: 15 November 2021 Accepted: 12 December 2021

Published online: 15 February 2022

\section{References}

1. Chaffer, C. L. \& Weinberg, R. A. A perspective on cancer cell metastasis. Science 331, 1559-1564 (2011).

2. Paterlini-Brechot, P. \& Benali, N. L. Circulating tumor cells (CTC) detection: clinical impact and future directions. Cancer Lett. 253, 180-204 (2007).

3. Sharma, S. et al. Circulating tumor cell isolation, culture, and downstream molecular analysis. Biotechnol. Adv. 36, 1063-1078 (2018).

4. Khetani, S., Mohammadi, M. \& Nezhad, A. S. Filter-based isolation, enrichment, and characterization of circulating tumor cells. Biotechnol. Bioeng. 115 2504-2529 (2018)

5. Kelley, S. O. \& Pantel, K. A new era in liquid biopsy: from genotype to phenotype. Clin. Chem. 66, 89-96 (2019).

6. Pei, H., Li, L., Han, Z., Wang, Y. \& Tang, B. Recent advances in microfluidic technologies for circulating tumor cells: enrichment, single-cell analysis, and liquid biopsy for clinical applications. Lab. Chip 20, 3854-3875 (2020).

7. Cohen, S. J. et al. Relationship of circulating tumor cells to tumor response, progression-free survival, and overall survival in patients with metastatic colorectal cancer. Clin. Oncol. 26, 3213-3221 (2008).

8. Riethdorf, S. et al. Detection and HER2 expression of circulating tumor cells: prospective monitoring in breast cancer patients treated in the neoadjuvant GeparQuattro trial. Clin. Cancer Res. 16, 2634-2645 (2010).

9. Lin, Z. et al. Recent advances in microfluidic platforms applied in cancer metastasis: circulating tumor cells' (CTCs) isolation and tumor-on-a-chip. Small 16, 1903899 (2020).

10. Park, E. S. et al. Continuous flow deformability-based separation of circulating tumor cells using microfluidic ratchets. Small 12, 1909-1919 (2016).

11. Chen, L. et al. Biofunctionalized magnetic nanospheres-based cell sorting strategy for efficient isolation, detection and subtype analyses of heterogeneous circulating hepatocellular carcinoma cells. Biosens. Bioelectron. 85, 633-640 (2016)

12. Chen, Y. et al. A new analytical platform for potential point-of-care testing of circulating tumor cells. Biosens. Bioelectron. 171, 112718-112718 (2021).

13. Min, H., Jo, S. \& Kim, H. Efficient capture and simple quantification of circulating tumor cells using quantum dots and magnetic beads. Small 11 2536-2542 (2015).

14. Yu, X. et al. Magneto-controllable capture and release of cancer cells by using a micropillar device decorated with graphite oxide-coated magnetic nanoparticles. Small 9, 3895-3901 (2013).

15. Kwak, B. et al. Spiral shape microfluidic channel for selective isolating of heterogenic circulating tumor cells. Biosens. Bioelectron. 101, 311-316 (2018).

16. Hyun, K. A., Lee, T. Y., Lee, S. H. \& Jung, H. I. Two-stage microfluidic chip for selective isolation of circulating tumor cells (CTCS). Biosens. Bioelectron. 67, 86-92 (2015).

17. Deliorman, M. et al. AFM-compatible microfluidic platform for affinity-based capture and nanomechanical characterization of circulating tumor cells. Microsyst. Nanoeng. 6, 1-15 (2020).

18. Chen, Y. et al. Rare cell isolation and analysis in microfluidics. Lab Chip 14, 626-645 (2014).

19. De Bono, J. S. et al. Circulating tumor cells predict survival benefit from treatment in metastatic castration-resistant prostate cancer. Clin. Cancer Res. 14, 6302-6309 (2008).

20. Hayes, D. F. et al. Circulating tumor cells at each follow-up time point during therapy of metastatic breast cancer patients predict progression-free and overall survival. Clin. Cancer Res. 12, 4218-4224 (2006).

21. Thiery, J. P. Epithelial-mesenchymal transitions in tumour progression. Nat. Rev. Cancer 2, 442-454 (2002).
22. Wu, T. H., Wu, C. H., Huang, C. J. \& Chang, Y. C. Anticlogging hemofiltration device for mass collection of circulating tumor cells by ligand-free size selection. Langmuir 37, 3399-3409 (2021).

23. Sun, N., Li, X., Wang, Z., Li, Y. \& Pei, R. High-purity capture of CTCs based on micro-beads enhanced isolation by size of epithelial tumor cells (ISET) method. Biosens. Bioelectron. 102, 157-163 (2018).

24. Gurudatt, N. G. et al. Separation detection of different circulating tumor cells in the blood using an electrochemical microfluidic channel modified with a lipid-bonded conducting polymer. Biosens. Bioelectron. 146, 111746 (2019).

25. Shen, $\mathrm{H}$. et al. A novel label-free and reusable electrochemical cytosensor for highly sensitive detection and specific collection of CTCs. Biosens. Bioelectron. 81, 495-502 (2016).

26. Turcan, I. \& Olariu, M. A. Dielectrophoretic manipulation of cancer cells and their electrical characterization. ACS Comb. Sci. 22, 554-578 (2020).

27. Li, M. \& Anand, R. K. High-throughput selective capture of single circulating tumor cells by dielectrophoresis at a wireless electrode array. J. Am. Chem. Soc. 139, 8950-8959 (2017).

28. Zhou, J. et al. Isolation of circulating tumor cells in non-small-cell-lung-cancer patients using a multi-flow microfluidic channel. Microsyst. Nanoeng. 5, 1-12 (2019).

29. Zhou, J., Peng, Z. \& Papautsky, I. Mapping inertial migration in the cross section of a microfluidic channel with high-speed imaging. Microsyst. Nanoeng. 6 1-14 (2020).

30. Rushton, A. J., Nteliopoulos, G., Shaw, J. A. \& Coombes, R. C. A review of circulating tumour cell enrichment technologies. Cancers 13, 970 (2021).

31. Harouaka, R. A. et al. Flexible micro spring array device for high-throughput enrichment of viable circulating tumor cells. Clin. Chem. 60, 323-333 (2014).

32. Desitter, I. et al. A new device for rapid isolation by size and characterization of rare circulating tumor cells. Anticancer Res. 31, 427-441 (2011).

33. Meunier, A. et al. Combination of mechanical and molecular filtration for enhanced enrichment of circulating tumor cells. Anal. Chem. 88, 8510-8517 (2016).

34. Sajay, B. N. et al. Microfluidic platform for negative enrichment of circulating tumor cells. Biomed. Microdevices 16, 537-548 (2014).

35. Hofman, $\mathrm{V}$. et al. Detection of circulating tumor cells as a prognostic factor in patients undergoing radical surgery for non-small-cell lung carcinoma: comparison of the efficacy of the CellSearch Assay ${ }^{\mathrm{TM}}$ and the isolation by size of epithelial tumor cell method. Int. J. Cancer 129, 1651-1660 (2011).

36. Farace, F. et al. A direct comparison of CellSearch and ISET for circulating tumour-cell detection in patients with metastatic carcinomas. Br. J. Cancer 105, 847-853 (2011).

37. Lin, H. K. et al. Portable filter-based microdevice for detection and characterization of circulating tumor cells. Clin. Cancer Res. 16, 5011-5018 (2010).

38. Tang, Y. D. et al. Microfluidic device with integrated microfilter of conicalshaped holes for high efficiency and high purity capture of circulating tumor cells. Sci. Rep. 4, 1-7 (2014).

39. Kim, T. H. et al. FAST: Size-selective, clog-free isolation of rare cancer cells from whole blood at a liquid-liquid interface. Anal. Chem. 89, 1155-1162 (2017).

40. Adams, D. L. et al. The systematic study of circulating tumor cell isolation using lithographic microfilters. RSC Adv. 4, 4334-4342 (2014).

41. Hosokawa, M. et al. Microcavity array system for size-based enrichment of circulating tumor cells from the blood of patients with small-cell lung cancer. Anal. Chem. 85, 5692-5698 (2013).

42. Sajay, B. N. G. et al. Towards an optimal and unbiased approach for tumor cell isolation. Biomed. Microdevices 15, 699-709 (2013).

43. Xu, T., Lu, B., Tai, Y. C. \& Goldkorn, A. A cancer detection platform which measures telomerase activity from live circulating tumor cells captured on a microfilter. Cancer Res. 70, 6420-6426 (2010).

44. Chen, J. J. \& Li, K. T. Analysis of PCR kinetics inside a microfluidic DNA amplification system. Micromachines 9, 48 (2018).

45. Rabe, M., Verdes, D. \& Seeger, S. Understanding protein adsorption phenomena at solid surfaces. Adv. Colloid Interface Sci. 162, 87-106 (2011).

46. Yusko, E. C. et al. Controlling protein translocation through nanopores with bio-inspired fluid walls. Nat. Nanotechnol. 6, 253-260 (2011).

47. Dong, $X$. et al. Coating magnetic nanospheres with PEG To reduce nonspecific adsorption on cells. ACS Omega 4, 7391-7399 (2019).

48. Fan, X. et al. A microfluidic chip integrated with a high-density PDMS-based microfiltration membrane for rapid isolation and detection of circulating tumor cells. Biosens. Bioelectron. 71, 380-386 (2015). 